\title{
Suspension Dosage Form Category
}

National Cancer Institute

\section{Source}

National Cancer Institute. Suspension Dosage Form Category. NCI Thesaurus. Code C154602.

A type of liquid pharmaceutical dose form consisting of one or more substances dispersed in a liquid vehicle; the dispersed substance(s) are solid particles that are practically insoluble in the vehicle. 